\title{
Snare combined with endoscopic clips in endoscopic resection of gastric submucosal tumor: a method of tumor traction $\square$
}

\section{(ㄷ)(1)}

Authors

Qiang Zhang, Jian-qun Cai, Zhen Wang, Bing Xiao, Yang Bai

Institution

Guangdong Provincial Key Laboratory of Gastroenterology, Department of Gastroenterology, Nanfang Hospital, Southern Medical University, Guangzhou, Guangdong Province, China

submitted 26.10.2018

accepted after revision $\quad 27.12 .2018$

\section{Bibliography}

DOI https://doi.org/10.1055/a-0849-9625 |

Endoscopy International Open 2019; 07: E1150-E1162

(c) Georg Thieme Verlag KG Stuttgart · New York eISSN 2196-9736

\section{Corresponding author}

Qiang Zhang, Guangdong Provincial Key Laboratory of Gastroenterology, Department of Gastroenterology, Nanfang Hospital, Southern Medical University, Guangzhou, Guangdong Province 510515, China Fax: +86-20-87280770

4024313@qq.com

\section{ABSTRACT}

Background and study aims Mucosal traction as a supportive technique is very useful for endoscopists during endoscopy. For gastric submucosal tumor (SMT), our team explored a method of pulling the SMT with a snare combined with endoclips (PSMT-SE). This study preliminarily explored its feasibility to assist resection of gastric SMT. Patients and methods Operation-related data from patients who underwent gastric SMT removal assisted by PSMT-SE at the Gastrointestinal Endoscopy Center of Guangzhou Nanfang Hospital, China between January 2017 and October 2018 were retrospectively collected: tumor size and location, origin of tumor, total operation time, en bloc resection rate, intraoperative and postoperative complications.

Results Forty-two gastric SMTs in 41 patients were included in this study. Fifteen tumors were located in the gastric fundus, 11 in the gastric body, two in the gastric angle, 10 in the gastric antrum, and four in the greater curvature of the gastric fundus and the body junction. Further, 11 tumors originated from the submucosa and 31 originated from the muscularis propria. Endoscopic submucosal dissection and endoscopic full-thickness resection assisted by PSMT-SE were performed to resect 30 and 12 tumors, respectively. PSMT-SE could effectively expose the surgical field. Median diameter of resected tumors was 2.0 (0.7) $\mathrm{cm}$, the total operation time was 45.5 (27.0) min, and the en bloc resection rate was $100 \%$. No intraoperative or postoperative complications were observed.

Conclusion PSMT-SE is a potentially useful method for assisting resection of gastric SMT with tumor traction. Further prospective studies with large sample sizes are warranted.

\section{Introduction}

Minimally invasive approaches may form the mainstay of surgical treatment for small submucosal tumors (SMT) [1]. Recently, endoscopic technology has rapidly developed. In some countries, especially in Asia, endoscopic treatment of gastric SMT is increasingly being used, suggesting that endoscopic resection of relatively small gastric SMTs is feasible and safe [2-7].

Gastric mucosal traction is known to effectively assist resection of gastric epithelial neoplasia [8,9]. Therefore, it is worthwhile to investigate the feasibility of using gastric SMT traction for assistance with tumor resection. Our team explored a method of endoscopic gastric SMT resection assisted by pulling the
SMT with a snare combined with endoclips (PSMT-SE). This method was expected to effectively pull the tumor to fully expose the endoscopic surgical field. It is crucial to pull the tumor toward the gastral cavity to reduce risk of damage to the abdominal organs and blood vessels by the endoscopic knife and falling of the tumor into the abdominal cavity in endoscopic full-thickness resection (EFR) [10]. In this study, our objective was to preliminarily explore the feasibility and safety of PSMTSE to assist resection of gastric SMT. 


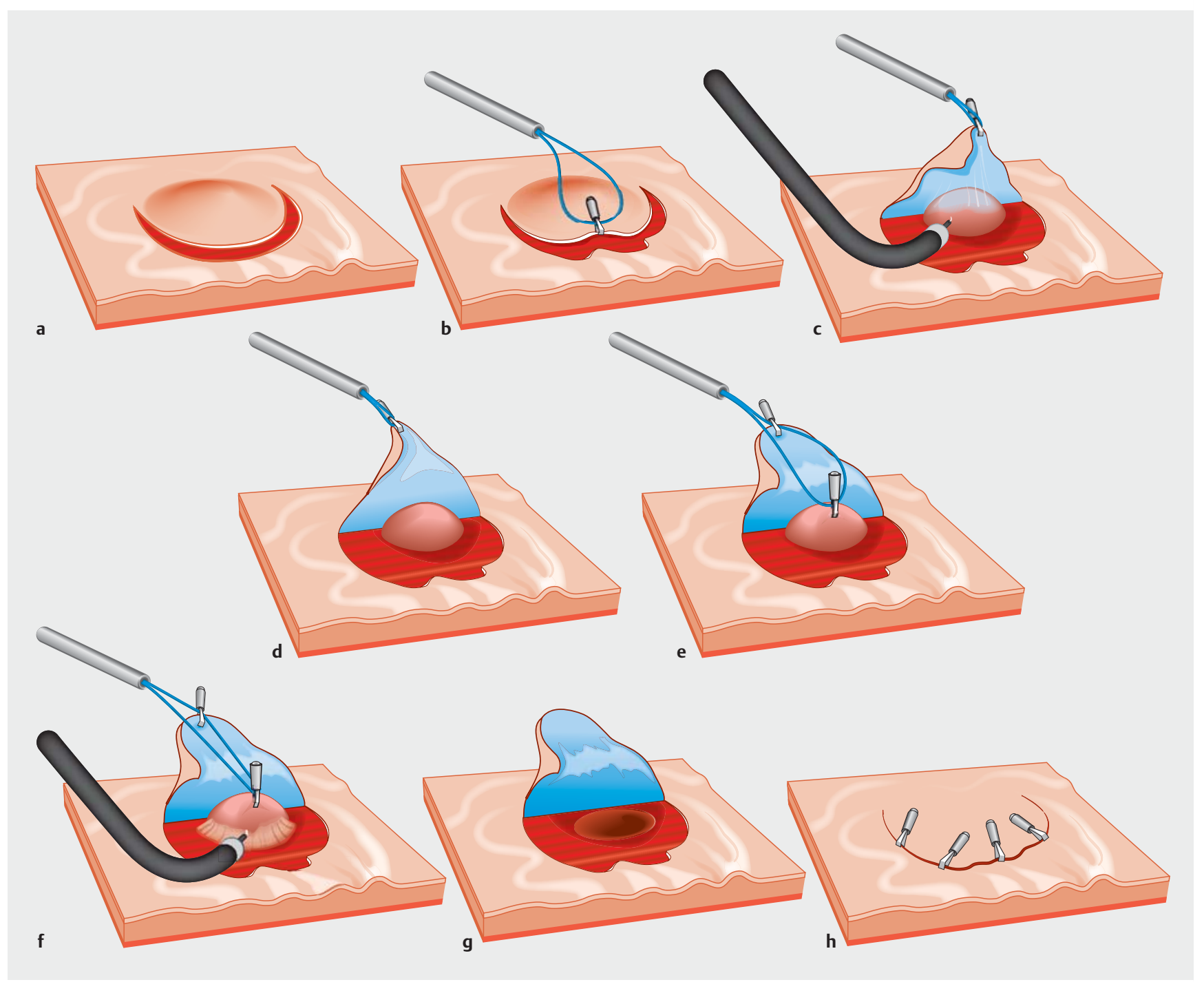

- Fig. 1 Flowchart of mucosa-sparing peroral external traction in removal of submucosal tumor (SMT). a Gastric mucosa is cut along the edge of the tumor for half to three quarters of the circumference. $\mathbf{b}$ The snare is fixed to the incised mucosal flap with endoclips. $\mathbf{c}$ The snare is pulled outside the body to pull the flap to expose the submucosa. Thereafter, the submucosa is dissected; $\mathbf{d}$ The tumor is fully exposed. e Further, the snare is fixed to the tumor using endoclips. $\mathbf{f}$ The snare is pulled to lift the tumor to expose the surgical field for tumor resection, and then, the tumor is dissected. $\mathbf{g}$ The wound. $\mathbf{h}$ The wound is sutured with the retained gastric mucosal flap combined with endoclips.

\section{Patients and methods}

From January 2017 to October 2018, 42 gastric SMTs in 41 consecutive patients were resected with the aid of PSMT-SE at the Gastrointestinal Endoscopy Center, Guangzhou Nanfang Hospital, China. Inclusion criteria were as follows: 1. tumor size $\geq 1 \mathrm{~cm}$, assessed using endoscopic ultrasonography; 2 . no high-risk EUS features, such as ulceration, irregular borders, internal heterogeneity, and regional lymph node enlargement; and 3.ESD or EFR performed for the removal of tumor. The following data were retrospectively collected: submucosal tumor location; long and short diameters of the tumor specimen directly measured postoperatively using a ruler; origin of the tumor including the following aspects: submucosa, muscularis propria, extraluminal and intraluminal growth assessed using endoscopic ultrasonography; operative time calculated from time of submucosal injection to complete resection; en bloc resection rate; intraoperative and postoperative complications; pathology of resected tumor; operative expense; and postoperative hospital stay. All the operations were performed by Dr Qiang Zhang who is experienced in ESD operation.

The main endoscopic accessories included the following: Endoscopes (GIF-QF260]; Olympus, Japan), Hook knife (KD-620LR; Olympus, Tokyo, Japan), IT knife (KD-611L; Olympus, Tokyo, Japan), Snare with maximum insertion diameter of $1.8 \mathrm{~mm}$ (SD221L-25; Olympus, Japan), and Hemoclips (HXROCC-D-26195-C, MICRO-TECH, China; HX-610-090 L, Olympus, Tokyo, Japan; M00522610, Boston Scientific Corporation, United States). Hemoclips that can be repeatedly opened and closed were the first choice because they can easily be manipulated to clamp the snare. 


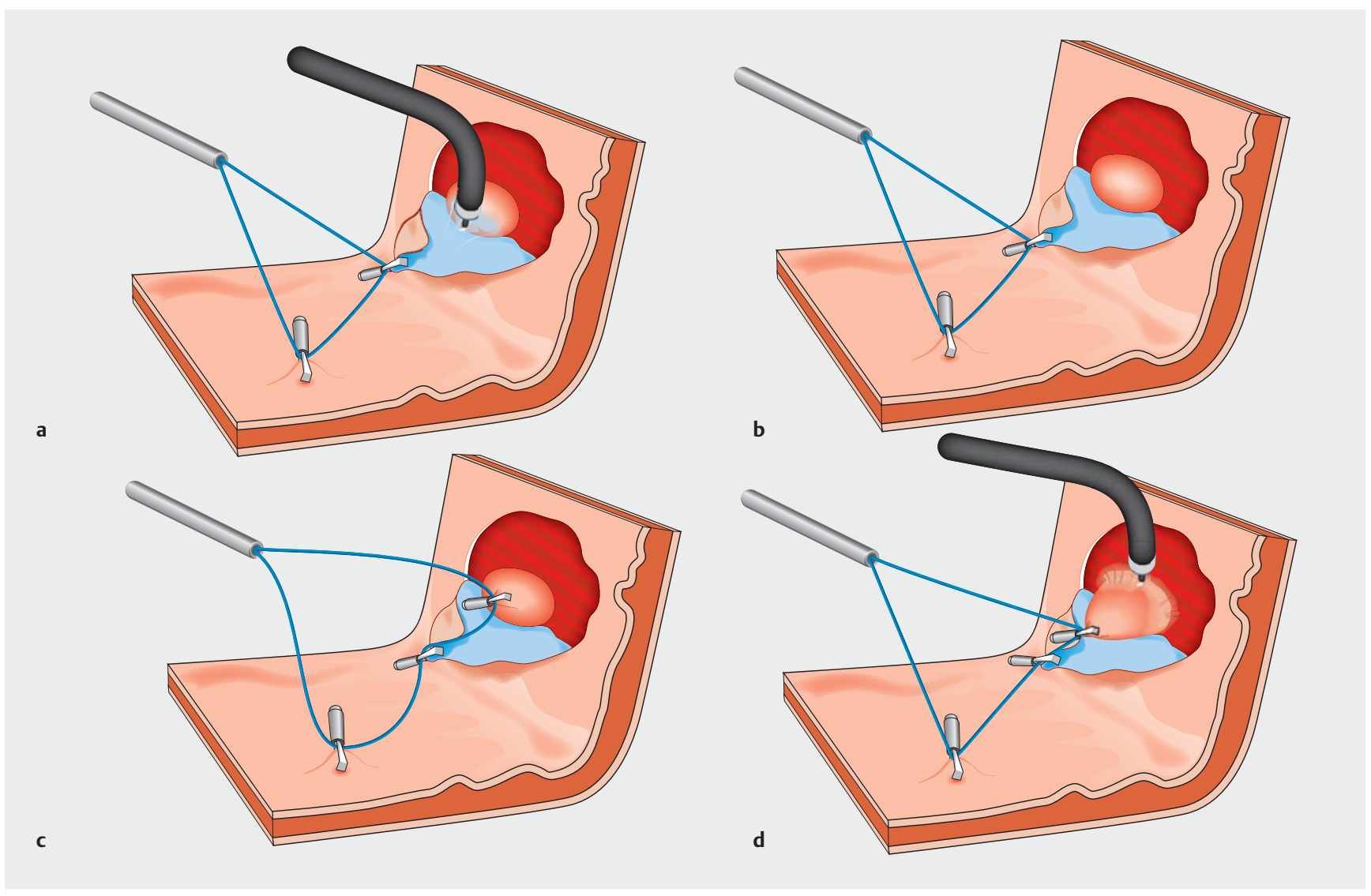

- Fig. 2 Key steps for mucosa-sparing peroral internal traction to assist tumor removal. a The snare is fixed to the incised mucosal flap using endoclips (a fixed site), and the snare is also fixed to the normal gastric mucosa opposite to the flap (the other fixed site). The snare is appropriately tightened to achieve the mutual pulling of the two fixed sites to expose the submucosa, and then, the submucosa is dissected. $\mathbf{b}$ The tumor is fully exposed. $\mathbf{c}$ Further, the snare is fixed to the tumor. $\mathbf{d}$ Appropriate force is applied to tighten the snare to pull the tumor. Thereafter, the tumor is dissected.

For patients with tumors located in the upper part of the gastric body and in the gastric fundus and those $>70$ years, especially those with large tumors and scheduled to undergo EFR resection, tracheal intubation anesthesia was usually applied in the operation room of the endoscopy center, and intravenous anesthesia could be selected for other patients. Postoperatively, surgical patients were administered intravenous (IV) proton pump inhibitors (PPI) starting the day of the operation, with oral administration permitted 3 days thereafter without routine prophylactic antibiotics. Liquid diet was given on the third postoperative day. Therapeutic time of IV PPI was prolonged and antibiotics were adopted on the day of the operation for 3 to 5 days with fasting for 3 to 5 days in the case of a large wound, long operation time, or performance of EFR.

All patients preoperatively underwent endoscopic ultrasonography and were informed of the benefits and risks of the procedure. They provided written informed consent before the procedure. Data were collected in an anonymous manner.

\section{Procedures}

PSMT-SE includes the following two methods: gastric mucosasparing traction and non-gastric mucosa-sparing traction. As shown in the diagrammatic sketches ( $\triangleright$ Fig. 1 and $\triangleright$ Fig. 2 ), the operative procedures for gastric mucosa-sparing traction were as follows:

1. After submucosal injection, the gastric mucosa was cut open along the edge of the tumor, and the incision range was half to three quarters of the circumference ( $>$ Fig.1a). The guiding principle in scope selection for mucosal incision was complete exposure and dissociation of the tumor from the submucosa via submucosal dissection. 2. Under endoscopic guidance, a snare was delivered into the stomach ( $\triangleright$ Supplementary Fig. 1). 3. The incised mucosal flap was pulled by the snare to expose the submucosa. Thereafter, it was dissected until the SMT was fully exposed ( $\mathbf{F i g . 1 b}$, $\mathbf{F i g . 1 c}$, and $\triangleright$ Fig. 1d). Two methods of mucosal traction can be selectively used. Peroral external traction (PET): the snare is fixed to the mucosal flap with endoclips and is pulled outside the body to expose the submucosa ( $\triangleright$ Fig. 1b and $\triangleright$ Fig. 1c). Peroral internal traction (PIT) was used when PET could not effectively expose the submucosa: the snare was fixed to the mucosal flap (the first fixed site) and also fixed to the normal gastric mucosa opposite the flap (the second fixed site) ( $\boldsymbol{F}$ Fig.2a). Then, the snare was properly tightened so that the two fixed sites pulled each other to fully expose the submucosa ( $\triangleright$ Fig. 2a and $\triangleright$ Fig. 2 b). 


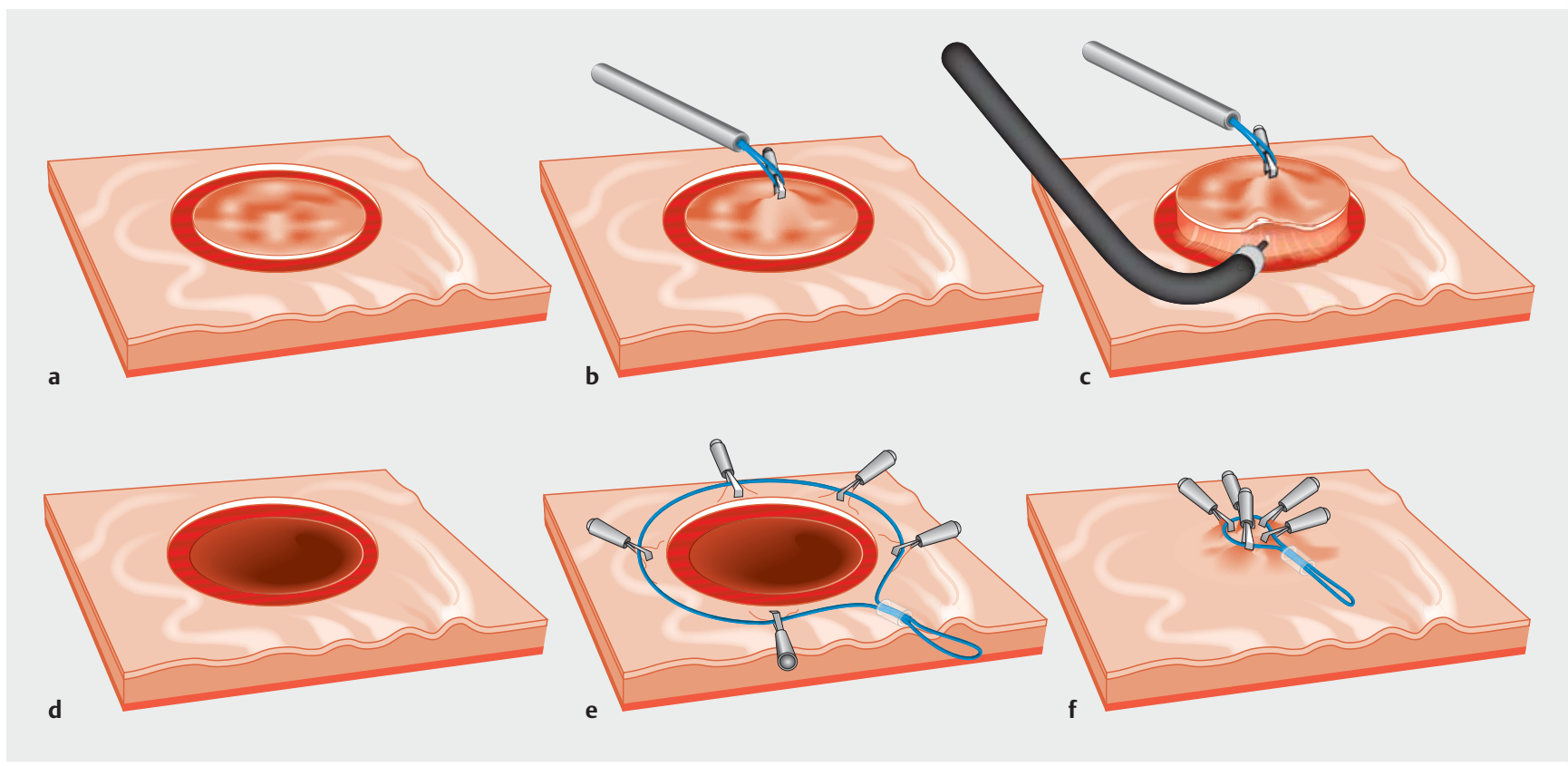

- Fig. 3 Flowchart of non-mucosa-sparing peroral external traction.a The gastric mucosa is cut open along the edge of the tumor. $\mathbf{b}$ The snare is fixed to the incised mucosa above the tumor using endoclips. c The snare is pulled outside the body to lift the tumor. Thereafter, the tumor is dissected. $\mathbf{d}$ The wound. e, f Purse-string suture is performed using a nylon thread combined with endoclips.

a

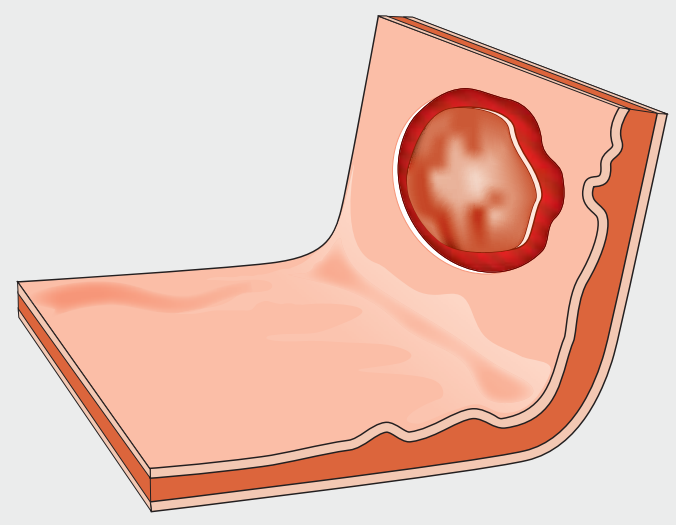

b

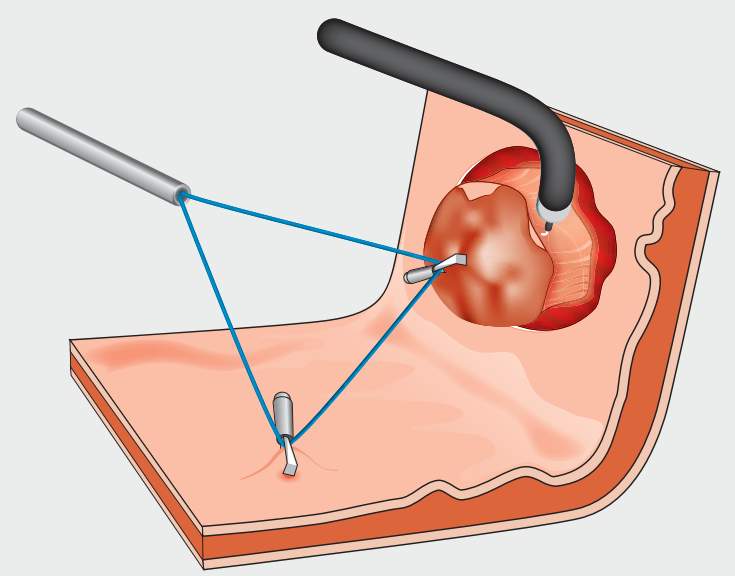

- Fig. 4 Key steps for non-mucosa-sparing peroral internal traction. a The gastric mucosa is cut open along the edge of the tumor. $\mathbf{b}$ The snare is fixed to the incised mucosa above the tumor using endoclips (a fixed site) and is fixed to the normal gastric mucosa opposite to the flap (the other fixed site). Thereafter, the snare is appropriately tightened to achieve the mutual pulling of the two fixed sites to lift the tumor. Thereafter, the tumor is dissected.

4. Further, the snare was directly fixed to the tumor, and then the tumor was pulled via PET ( $\triangleright$ Fig.1e and $\triangleright$ Fig. 1f) or PIT ( $\triangleright$ Fig. $\mathbf{2 c}$ and $\triangleright$ Fig. 2 d) to thoroughly expose the muscularis propria side of the tumor. Procedures for PET and PIT are similar to those described in the preceding paragraph. 5. After the tumor was completely removed, for EFR, suspicious perforation or prevention of delayed perforation, the wound was closed with the retained gastric mucosa combined with endoclips ( $\vee$ Fig. $\mathbf{1 g}$ and $\vee$ Fig. $\mathbf{1 h}$ ).
The other method was non-gastric mucosa-sparing traction ( $\vee$ Fig.3 and $\triangleright$ Fig.4). After incision of the gastric mucosa along the edge of the tumor ( $\triangleright$ Fig. 3a), the snare was directly fixed to the incised mucosa above the tumor with endoclips ( $\vee$ Fig. 3b). Then, the tumor was pulled using PET and dissected ( $\vee$ Fig.3c). When PET could not effectively pull the tumor, PIT could be used ( $\vee$ Fig. 4). The PET and PIT procedures were similar to those described for gastric mucosa-sparing traction. For a large wound of tumor resection, especially perforation, it 
- Table 1 Summary of data from gastric submucosal tumors resected with the assistance of PSMT-SE.

\begin{tabular}{|c|c|c|}
\hline Patient/operation characteristics & Gastric mucosa-sparing traction & Non-gastric mucosa-sparing traction \\
\hline No. of patients & 20 & 22 \\
\hline Gender (Male/Female) & \multicolumn{2}{|l|}{$24 / 18$} \\
\hline Age, years (mean SD/range) & \multicolumn{2}{|l|}{$55.2(10.0),(28-73)$} \\
\hline \multicolumn{3}{|l|}{ Lesion sites ( $N$ ) } \\
\hline Gastric angle/ Gastric antrum/ Gastric body/ Gastric fundus & $0 / 4 / 7 / 8$ & $2 / 6 / 4 / 7$ \\
\hline Gastric fundus and body junction & 1 & 3 \\
\hline \multicolumn{3}{|l|}{ Origin of lesion $(\mathrm{N})$} \\
\hline Submucosa/ Muscularis propria & $0 / 16$ & $11 / 7$ \\
\hline Extraluminal/ Intraluminal growth & $4 / 0$ & $1 / 3$ \\
\hline \multicolumn{3}{|l|}{ Operation (N) } \\
\hline $\mathrm{ESD} / \mathrm{EFR}$ & $12 / 8$ & $18 / 4$ \\
\hline \multicolumn{3}{|l|}{ Traction types (N) } \\
\hline PET/PIT & $12 / 8$ & $14 / 8$ \\
\hline \multicolumn{3}{|l|}{ Methods of closing wound } \\
\hline RGM-E/ NT-E/Endoclip/ No closure & $13 / 3 / 0 / 4$ & $0 / 3 / 8 / 11$ \\
\hline Operative time for all tumors, min (median IQR/SD) & \multicolumn{2}{|l|}{$45.5(27.0)(19-76)$} \\
\hline Lesion size for all tumors, $\mathrm{cm}$ (median IQR/SD) & \multicolumn{2}{|l|}{$2.0(0.7)(1.0-3.0)$} \\
\hline Operation time, min (median IQR/SD) & 46.0 (20.5), $(31-76)$ & $43.5(33.0),(19-67)$ \\
\hline Lesion size in long diameter, $\mathrm{cm}$ (median IQR/SD) & $2.0(0.5),(1.2-2.5)$ & $1.8(1.2),(1.0-3.0)$ \\
\hline Lesion size in short diameter, cm (median IQR/SD) & $1.5(1.0),(1.0-2.0)$ & $1.5(1.0),(1.0-3.0)$ \\
\hline En bloc resection rate (\%) & $100 \%$ & $100 \%$ \\
\hline \multicolumn{3}{|l|}{ Pathology (N) } \\
\hline Stromal tumor, very low/low risk & $9 / 4$ & $6 / 4$ \\
\hline leiomyoma/ectopic pancreas/others & $4 / 1 / 2$ & $3 / 4 / 5$ \\
\hline Operation cost, USD (mean [SD]/range) & $2159.0(600.5)$ & $2137.1(432.0)$ \\
\hline Postoperative hospital days, days (mean [SD]/range) & $6(2)$ & $6(2)$ \\
\hline \multicolumn{3}{|l|}{ Postoperative complications (N) } \\
\hline Delayed bleeding/perforation & 0 & 0 \\
\hline
\end{tabular}

might be necessary to use a nylon thread with endoclips to perform the purse-string suture ( $>$ Fig. 3 e and $>$ Fig. $\mathbf{3 f}$ ).

Generally speaking, standards for choosing gastric mucosasparing traction or non-gastric mucosa-sparing traction were as follows. In general, for SMTs that were relatively flat and originated from the muscularis propria, especially those presenting with an extraluminal growth and those with a high possibility of perforation or full-thickness resection as per the preoperative assessment, mucosa-sparing traction was used. For SMTs located in the submucosa or those obviously bulging into the gastric cavity with difficulty in retaining the gastric mucosa over the tumor, non-mucosa-sparing traction was used. Moreover, the standards for choosing PET or PIT were as follows: first, consider the use of PET; when the traction effect of PET was poor, PIT could be chosen.

\section{Statistical analyses}

Data were expressed as mean (standard deviation) and median (interquartile range $[I Q R]=$ the third quartile [Q3]-the first quartile [Q1]) values. Data were analyzed using Microsoft Excel. 


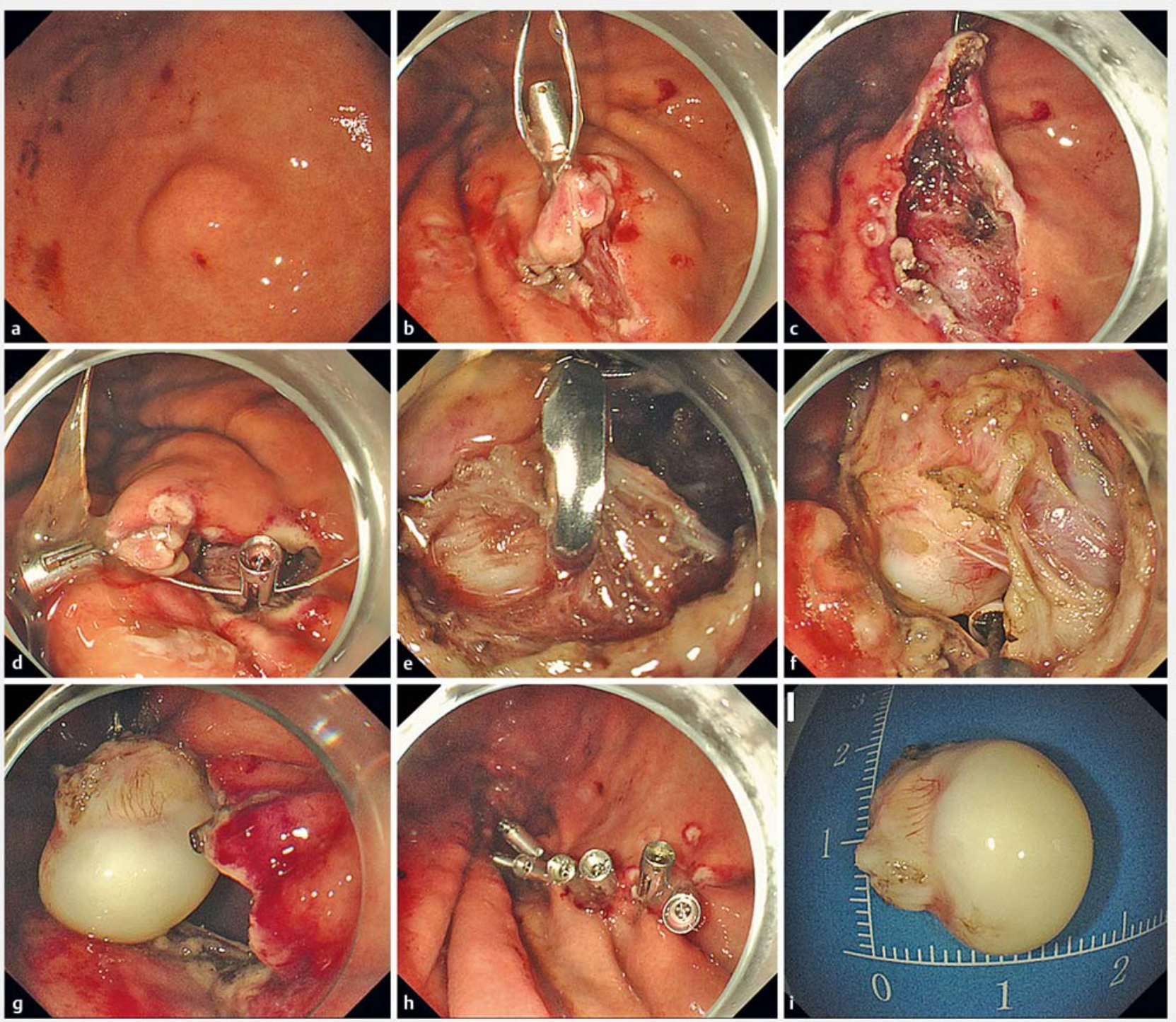

- Fig. 5 EFR assisted by PSMT-SE for resection of a submucosal tumor at the gastric body. Mucosa-sparing peroral external traction was used. a A tumor at the gastric body. $\mathbf{b}$ The snare is fixed to the incised mucosal flap using endoclips. $\mathbf{c}$ The snare is pulled to expose the submucosa, and the submucosa is dissected till the tumor is fully exposed. $\mathbf{d}$ Further, the snare is fixed to the tumor. e, $\mathbf{f}, \mathbf{g}$ The snare is pulled to lift the tumor to expose the surgical field. Thereafter, the tumor is dissected. $\mathbf{h}$ The perforation is sutured with the retained gastric mucosal with endoclips. $\mathbf{i}$ The resected tumor. The case shown in the $\mathbf{F i g .} \mathbf{5}$ is the same as that shown in $\mathbf{V i d e o} \mathbf{1}$

\section{Results}

In this study, 42 gastric SMTs in 41 patients were included. A total of 15 tumors were located in the gastric fundus, 11 in the gastric body, two in the gastric angle, 10 in the gastric antrum, and four in the greater curvature of the gastric fundus and the body junction. Further, 11 tumors originated from the submucosa and 31 originated from the muscularis propria. ESD and EFR, with the aid of PSMT-SE, were performed to resect 30 and 12 tumors, respectively.

PSMT-SEs were performed in the 42 cases that included 20 cases in which gastric mucosa-sparing traction was used and 22 cases in which non-gastric mucosa-sparing traction was used. In 20 cases of gastric mucosa-sparing traction, PET and
PIT were used in 12 and 8 cases, respectively. In 22 cases of non-gastric mucosa-sparing traction, PET and PIT were used in 14 and 8 cases, respectively.

PSMT-SE can effectively pull the gastric mucosa and SMT to fully expose the surgical field. In EFR, with the aid of PSMT-SE, the tumor can be effectively pulled toward the gastric cavity, reducing risk of operation and preventing the tumor from falling into the abdominal cavity during resection. Median diameter of the resected tumors was $2.0(0.7) \mathrm{cm}$, operative time was 45.5 (27.0) $\mathrm{min}$, and the en bloc resection rate was $100 \%$. In cases with gastric mucosa-sparing traction, no tumor rupture caused due to direct clamping of the tumor with endoclips occurred, and the mucosal flap blood supply was reduced due to 

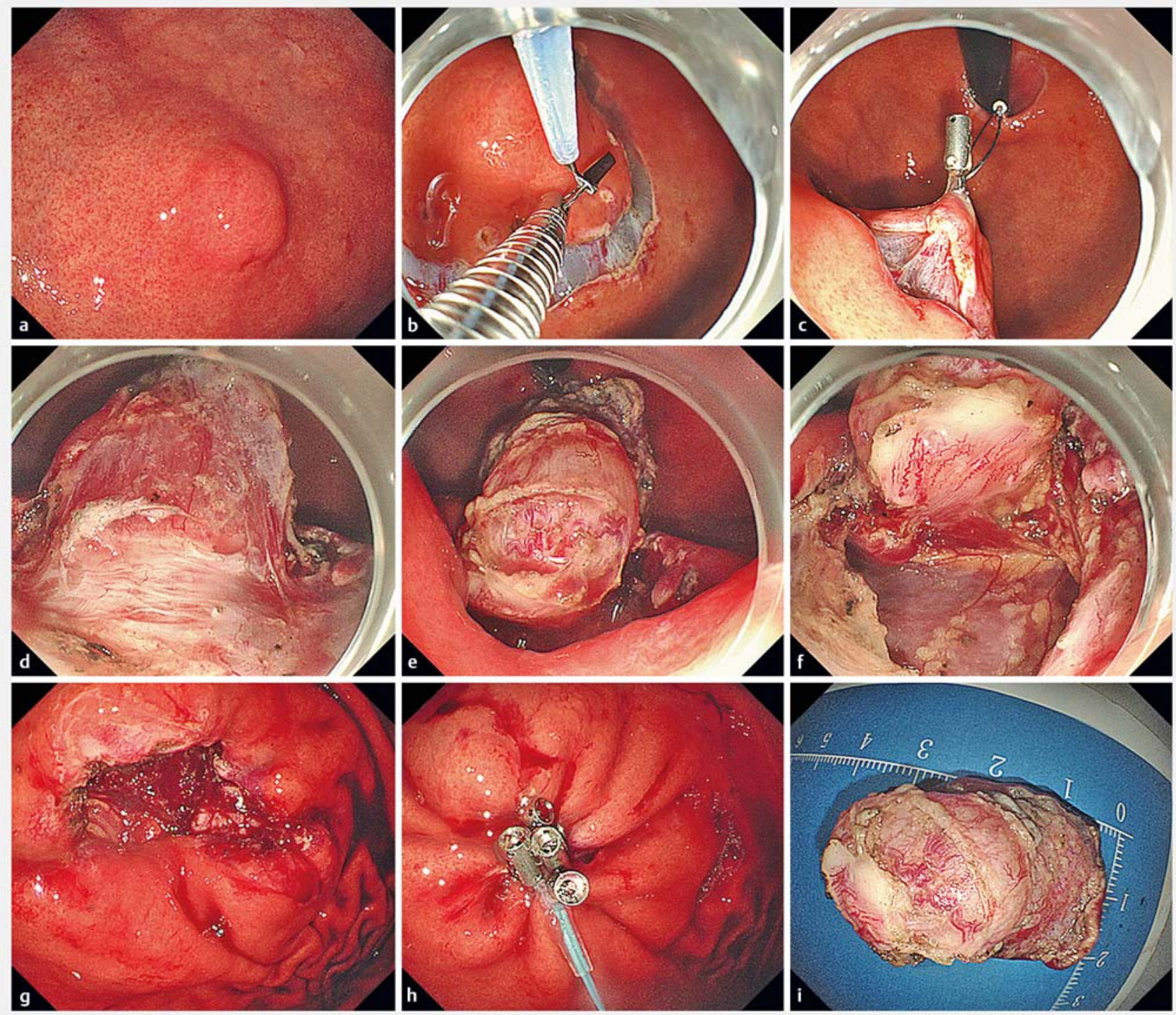

- Fig. 6 EFR assisted by PSMT-SE for resection of a submucosal tumor at the greater curvature of the upper part of the gastric body. Non-gastric mucosa-sparing peroral external traction was used. a A submucosal tumor. $\mathbf{b}$ The snare is fixed to the incised mucosa above the tumor using endoclips. $\mathbf{c}, \mathbf{d}, \mathbf{e}, \mathbf{f}$ The snare is pulled to lift the tumor toward the gastral cavity and then dissected. $\mathbf{g}$ The perforation. $\mathbf{h}$ Purse-string suture using a nylon thread with endoclips. i The resected tumor. The case shown in $\nabla$ Fig. $\mathbf{6}$ is the same as that shown in $\nabla$ Video $\mathbf{2}$.

mucosal incision. Intraoperative bleeding was effectively stopped without severe bleeding, and no perforation occurred during ESD. No postoperative delayed bleeding or perforation was observed.

All the data are shown in $\bullet$ Table 1 . Some practical examples are shown in $>$ Fig. 5, $>$ Fig. 6, and $\triangleright$ Fig. 7; $>$ Supplementary Fig. 2 and $\triangleright$ Supplementary Fig. 3; and $>$ Video 1 , $>$ Video 2 , $\checkmark$ Video 3 , and $\sim$ Video 4.

\section{Discussion}

In this study, PSMT-SE was able to fully expose the surgical field. In particular, the pulling effect could be adjusted in real time using the in vitro snare. A total of $42 \mathrm{SMTs} \geq 1 \mathrm{~cm}$ and $\leq 3 \mathrm{~cm}$ were safely and completely removed.

Currently, the main endoscopic methods for removal of SMTs $>1 \mathrm{~cm}$ are endoscopic submucosal dissection (ESD) [11, 12], submucosal tunneling endoscopic resection (STER) [13], and EFR [10]. Our team also explored use of endoscopic mucosa-sparing lateral dissection (EMSLD) $[14,15]$, which can be effectively used to remove gastric SMT.

Mucosal traction has been used to assist ESD for gastric epithelial neoplasia and can serve as a supportive technique to ef- 


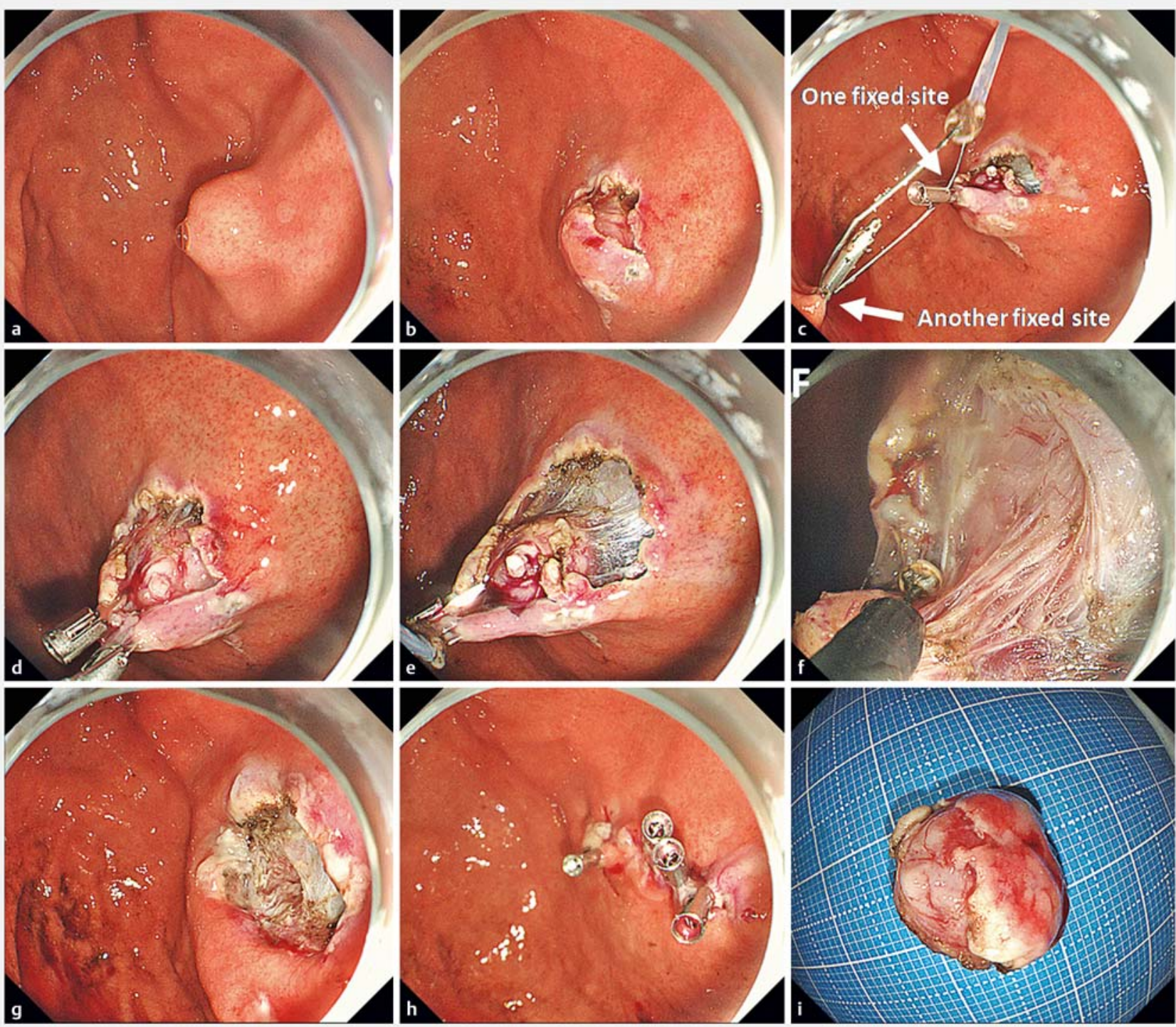

- Fig. 7 ESD assisted by PSMT-SE for the resection of a submucosal tumor at the gastric fundus. Non-gastric mucosa-sparing peroral internal traction was used. a A submucosal tumor. $\mathbf{b}$ Gastric mucosa is cut along the edge of the tumor. $\mathbf{c}$ The snare is fixed to the incised mucosa using endoclips (a fixed site) and was fixed to the normal gastric mucosa opposite to the flap (the other fixed site). d, e, f. Thereafter, the snare is tightened with appropriate force so that the two fixed sites pull each other to lift the tumor toward the gastral cavity. Thereafter, the tumor is dissected. $\mathbf{g}$ The wound. $\mathbf{h}$ The wound closed with endoclips. $\mathbf{i}$ The tumor. The case shown in $\nabla$ Fig. $\mathbf{7}$ is the same as that shown in $\nabla$ Video $\mathbf{3}$.

fectively pull the mucosa to expose the surgical field of the submucosa [9]. Few reports on removal of gastric SMT using ESD or EFR assisted with traction are currently available. Snare and endoclips are easily accessible at any endoscopy center. In this study, PSMT-SE was able to fully expose the surgical field to assist ESD or EFR for gastric SMTs. Li J et al. performed a study focusing on application of clip-with-thread for traction in gastric SMTs. The results revealed that tumor traction could effectively shorten operative time and improve operative safety [16]. However, further studies are required, considering that this study had a relatively small sample size. The clip-and-thread technique is the traction method most commonly used for assisting resection of gastric mucosal lesions. SMTs mostly origi- nate from the muscularis propria. Therefore, firmness and strength of traction with the clip-and-thread technique [17, 18] appear limited. In contrast, a snare in combination with endoclips is characterized by relatively great pulling force and flexible adjustability of the operation. In particular, PSMT-SE can achieve two types of PET and PIT. Moreover, within a certain range, traction force can be adjusted in real time by pulling the snare or adjusting the handle controller of the snare in vitro to fully expose the operative field of vision.

In this study, both gastric mucosa-sparing and non-gastric mucosa-sparing traction were explored. The former has the main advantage of EMSLD [14,15], i.e., the retained gastric mucosal flap helps repair the wound or perforation. Moreover, 
the snare is directly fixed to the tumor with endoclips. Thus, the force of pulling the tumor appears great; such a force could be preferable for pulling tumors that originate from the deep layer of the muscularis propria, especially those growing out of the gastric cavity. By comparison, the operation with non-gastric mucosa-sparing traction is relatively simple: the snare is fixed directly to the incised mucosa above the tumor. However, loose connective tissue in the submucosa lies between the tumor and the incised mucosa. Therefore, the pulling force of this traction seems relatively inefficient for tumors originating from the deep layer of the muscularis propria and those growing out of the gastric cavity. Moreover, a large wound or perforation needs to be repaired using a nylon thread or over-the-scope clip suture. For gastric mucosa-sparing and non-gastric mucosa-sparing traction, either PET or PIT can be used. PIT is relative-

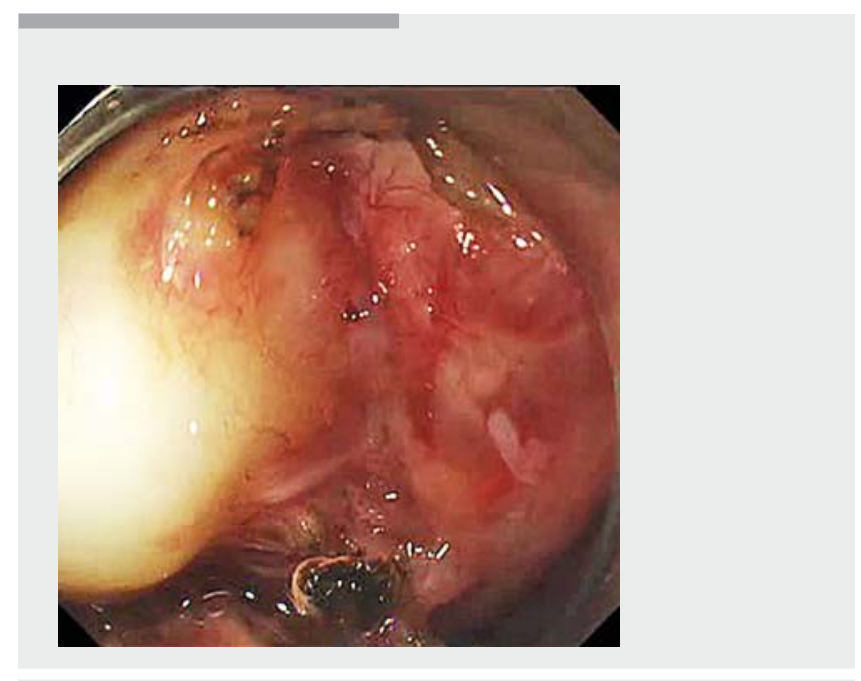

Video 1 EFR assisted by PSMT-SE (mucosa-sparing peroral external traction) for resection of a submucosal tumor at the gastric body.

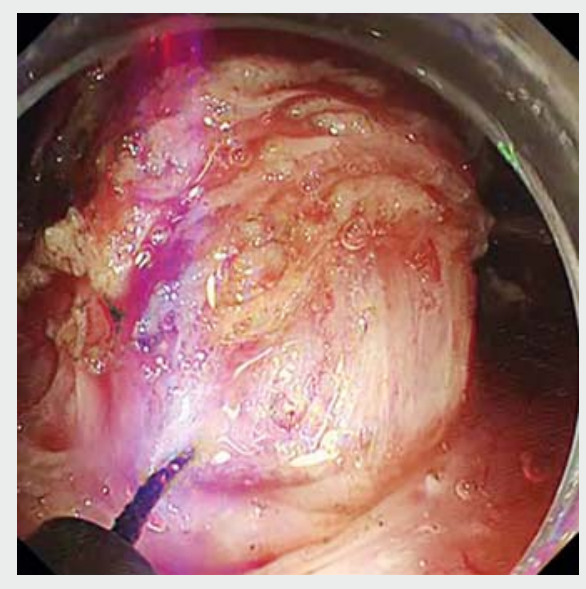

$\checkmark$ Video 2 EFR assisted by PSMT-SE (non-gastric mucosa-sparing peroral external traction) for resection of a submucosal tumor at the greater curvature of the upper part of the gastric body. ly complicated as compared to the procedure for external traction. Therefore, PET should be considered first. PET can only be directed toward the cardia, thus its traction effect is affected by tumor location. Under the circumstances, PIT can be used.

Several limitations of our method should be acknowledged. The endoclips attached to the gastric mucosa or tumor can break away if the pulling force is too great. To resolve this issue, multiple endoclips can be used to fix the snare to the target mucosa or the tumor to make the fixation firmer. Further, the type of endoclips can be chosen based on performance in terms of better force and firmness. During the operation, the snare we used was relatively thin and soft, resulting in relatively poorer effect of pushing to expose the surgical field of vision. Thus, we hypothesized that in theory, a thicker and harder snare would contribute to an improved effect of pushing. However,

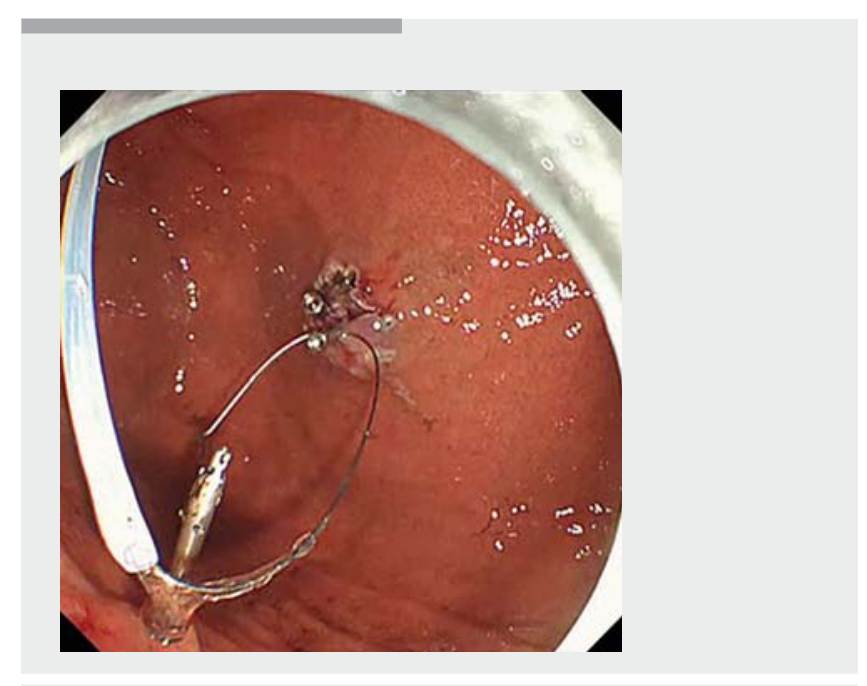

$\nabla$ Video 3 ESD assisted by PSMT-SE (non-gastric mucosa-sparing peroral internal traction) for resection of a submucosal tumor at the gastric fundus.

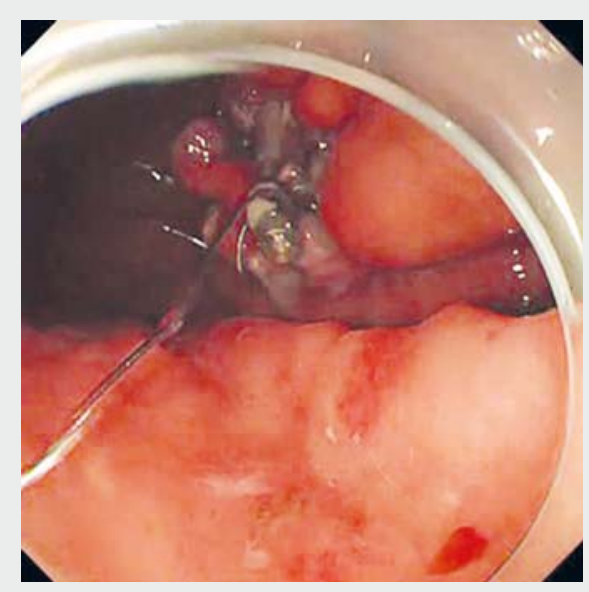

Video 4 EFR assisted by PSMT-SE (mucosa-sparing peroral internal traction) for resection of a submucosal tumor at the gastric fundus. 
its safety requires further evaluation. Currently, endoscopic resection of gastric SMTs involves a common problem, i.e., difficulty in achieving the same "non-contact resection" as that obtained in laparotomy and laparoscopy to prevent the endoscopic knife and hemostatic forceps from damaging the surface capsule of the tumor. Nonetheless, existing large-scale studies with long-term follow-up [4-7] have revealed that endoscopic resection seems to be feasible and safe for gastric SMTs that are relatively small, including stromal tumors. For mucosa-sparing traction-assisted EFR, endoclips were directly fixed to the surface of the tumor to perform traction. In this study, obvious defects in the tumor capsule and tissue caused by traction were not observed with the naked eye, but long-term follow-up is necessary to assess presence of peritoneal dissemination and metastasis.

This study had the following limitations. It employed a retrospective, single-arm design and the sample size was relatively small. It proposed two methods of traction (i.e., gastric mucosa-sparing and non-sparing methods), both of which have advantages and disadvantages. We also preliminarily set the general criteria for the choice between the two methods. To better choose a simple and effective traction method as per the different tumor characteristics, more operational experience is required.

\section{Conclusion}

In summary, PSMT-SE can effectively assist ESD and EFR for resection of gastric SMT with tumor traction. Further prospective studies involving a larger sample size are warranted.

\section{Competing interests}

\section{None}

\section{References}

[1] Nishida T, Kawai N, Yamaguchi S et al. Submucosal tumors: comprehensive guide for the diagnosis and therapy of gastrointestinal submucosal tumors. Dig Endosc 2013; 25: 479-489

[2] Cai MY, Martin Carreras F, Zhou PH. Endoscopic full-thickness resection for gastrointestinal submucosal tumors. Dig Endosc 2018; 30: $17-24$

[3] Abe N, Takeuchi H, Ohki A et al. Comparison between endoscopic and laparoscopic removal of gastric submucosal tumor. Dig Endosc 2018; 30: $7-16$
[4] Joo MK, Park J], Kim H et al. Endoscopic versus surgical resection of GI stromal tumors in the upper GI tract. Gastrointest Endosc 2016; 83: $318-326$

[5] Zhang Y, Mao XL, Zhou XB et al. Long-term outcomes of endoscopic resection for small $(\leq 4.0 \mathrm{~cm})$ gastric gastrointestinal stromal tumors originating from the muscularis propria layer. World J Gastroenterol 2018; $24: 3030-3037$

[6] Ye LP, Zhang Y, Luo DH et al. Safety of endoscopic resection for upper gastrointestinal subepithelial tumors originating from the muscularis propria layer: an analysis of 733 tumors. Am J Gastroenterol 2016; 111: $788-796$

[7] Park J]. Long-term outcomes after endoscopic treatment of gastric gastrointestinal stromal tumor. Clin Endosc 2016; 49: 232-234

[8] Tsuji K, Yoshida N, Nakanishi H et al. Recent traction methods for endoscopic submucosal dissection. World J Gastroenterol 2016; 22: $5917-5926$

[9] Fukami N. What we want for ESD is a second hand! Traction method Gastrointes Endosc 2013; 78: 274-276

[10] Zhou PH, Yao LQ, Qin XY et al. Endoscopic full-thickness resection without laparoscopic assistance for gastric submucosal tumors originated from the muscularis propria. Surg Endosc 2011; 25: 2926 2931

[11] Abe N, Takeuchi H, Ooki A et al. Recent developments in gastric endoscopic submucosal dissection: towards the era of endoscopic resection of layers deeper than the submucosa. Dig Endosc 201325: $64-70$

[12] Catalano F, Rodella L, Lombardo F et al. Endoscopic submucosal dissection in the treatment of gastric submucosal tumors: results from a retrospective cohort study. Gastric Cancer 2013; 16: 563-570

[13] Li QL, Chen WF, Zhang C et al. Clinical impact of submucosal tunneling endoscopic resection for the treatment of gastric submucosal tumors originating from the muscularis propria layer (with video). Surg Endosc 2015; 29: 3640 - 3646

[14] Zhang Q, Li Y, Meng Y et al. Should the integrity of mucosa be considered in endoscopic resection of gastric submucosal tumors? Gastroenterology 2016; 150: 822-4.e9

[15] Zhang Q, Li Y, Lian ZY et al. A modified endoscopic method for resection of gastric submucosal tumor. Surg Endosc 2018; 32: 536- 543

[16] Li J, Meng Y, Ye S et al. Usefulness of the thread-traction method in endoscopic full-thickness resection for gastric submucosal tumor: a comparative study. Surg Endosc 2018: doi:10.1007/s00464-0186585-2 [Epub ahead of print]

[17] Suzuki S, Gotoda T, Kobayashi Y et al. Usefulness of a traction method using dental floss and a hemoclip for gastric endoscopic submucosal dissection: a propensity score matching analysis (with videos). Gastrointest Endosc 2016; 83: 337-346

[18] Yoshida M, Takizawa K, Ono H et al. Efficacy of endoscopic submucosal dissection with dental floss clip traction for gastric epithelial neoplasia: a pilot study (with video). Surg Endosc 2016; 30: 3100-3106 


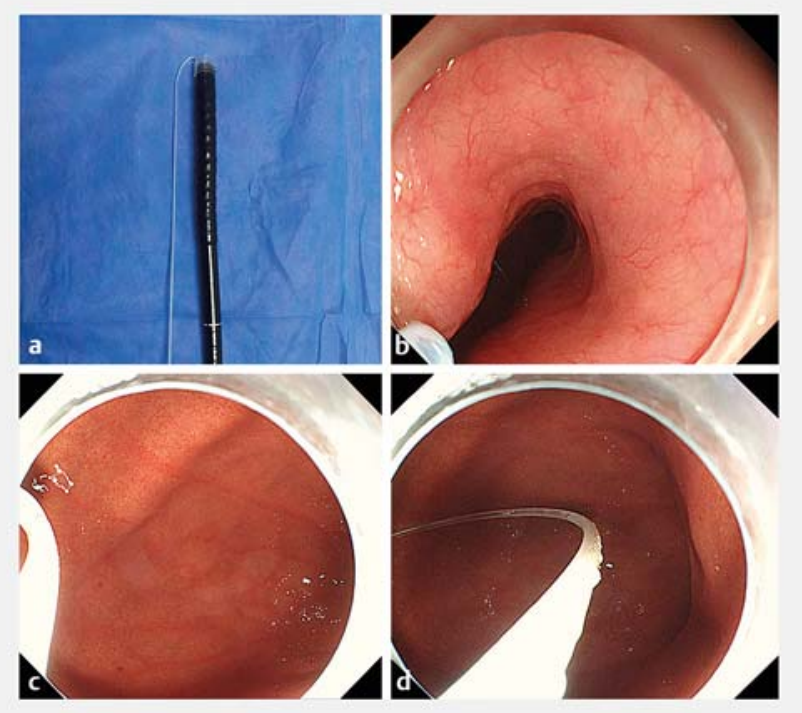

- Supplementary Fig. 1 A snare delivered into the stomach under endoscopic guidance. a A snare is inversely inserted from the head end of the endoscope into the endoscopic working channel.

$\mathbf{b}, \mathbf{c}$ Then, the snare is delivered into the stomach. $\mathbf{d}$ After entering the stomach, the snare is pushed out with an endoclip from the working channel. 

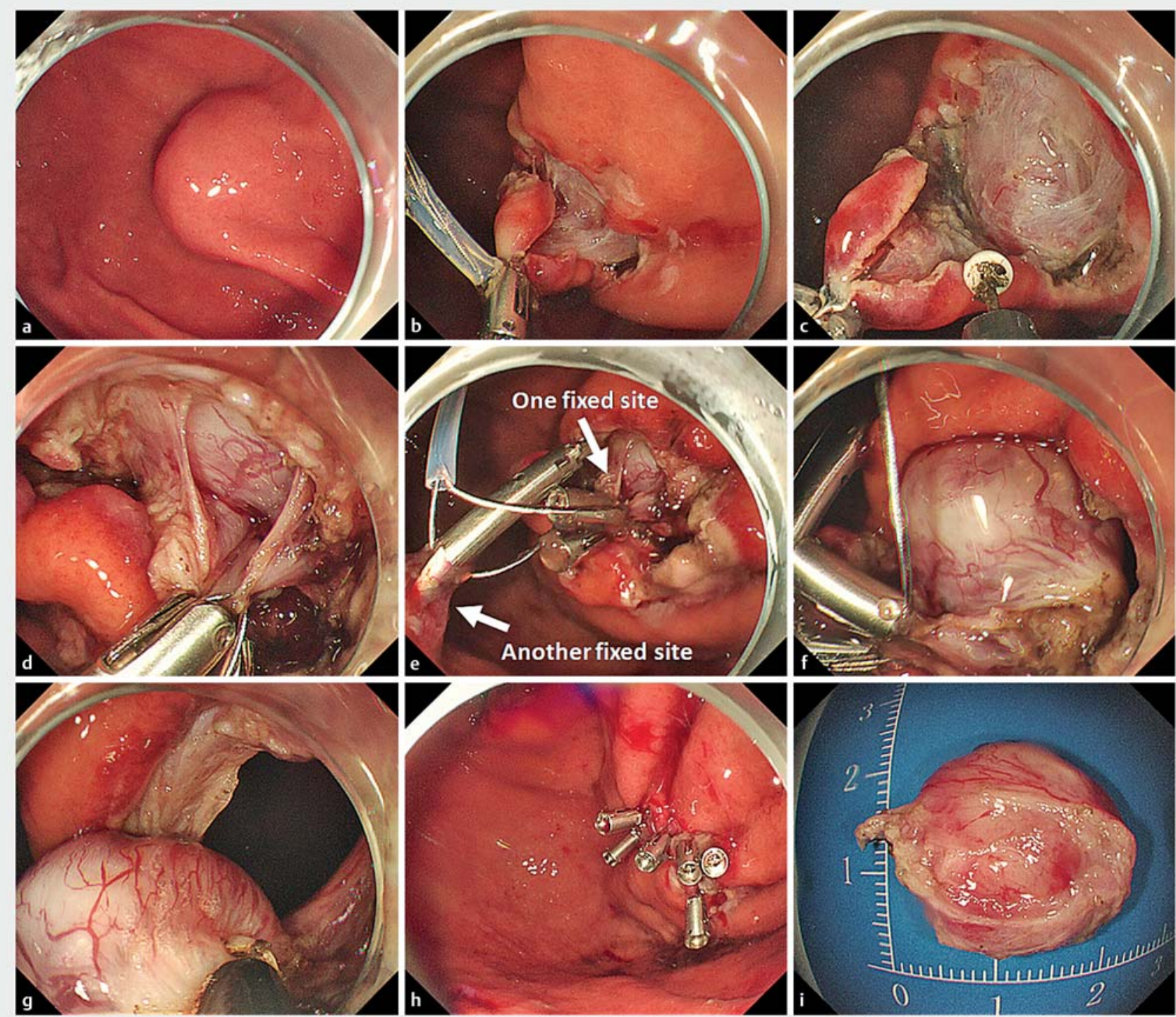

- Supplementary Fig. 2 EFR assisted by PSMT-SE for the resection of a submucosal tumor at the gastric fundus. Mucosa-sparing peroral internal traction was used. a Tumor. b, $\mathbf{c}$ The snare is fixed to the incised mucosal flap with endoclips and then pulled to expose the submucosa. The submucosa is dissected until the tumor is fully exposed, $\mathbf{d}$, e Further, the snare is fixed to the tumor (a fixed site) and to the normal gastric mucosa opposite to the flap (the other fixed site). $\mathbf{f}, \mathbf{g}$ The snare is tightened with appropriate force so that the two fixed sites pull each other to lift the tumor; thereafter, the tumor is dissected. $\mathbf{h}$ The perforation was sutured with the retained gastric mucosa with endoclips. $\mathbf{i}$ The resected tumor. The case shown in $\triangleright$ Supplementary Fig. 2 is the same as that shown in $\triangleright$ Video 4. 


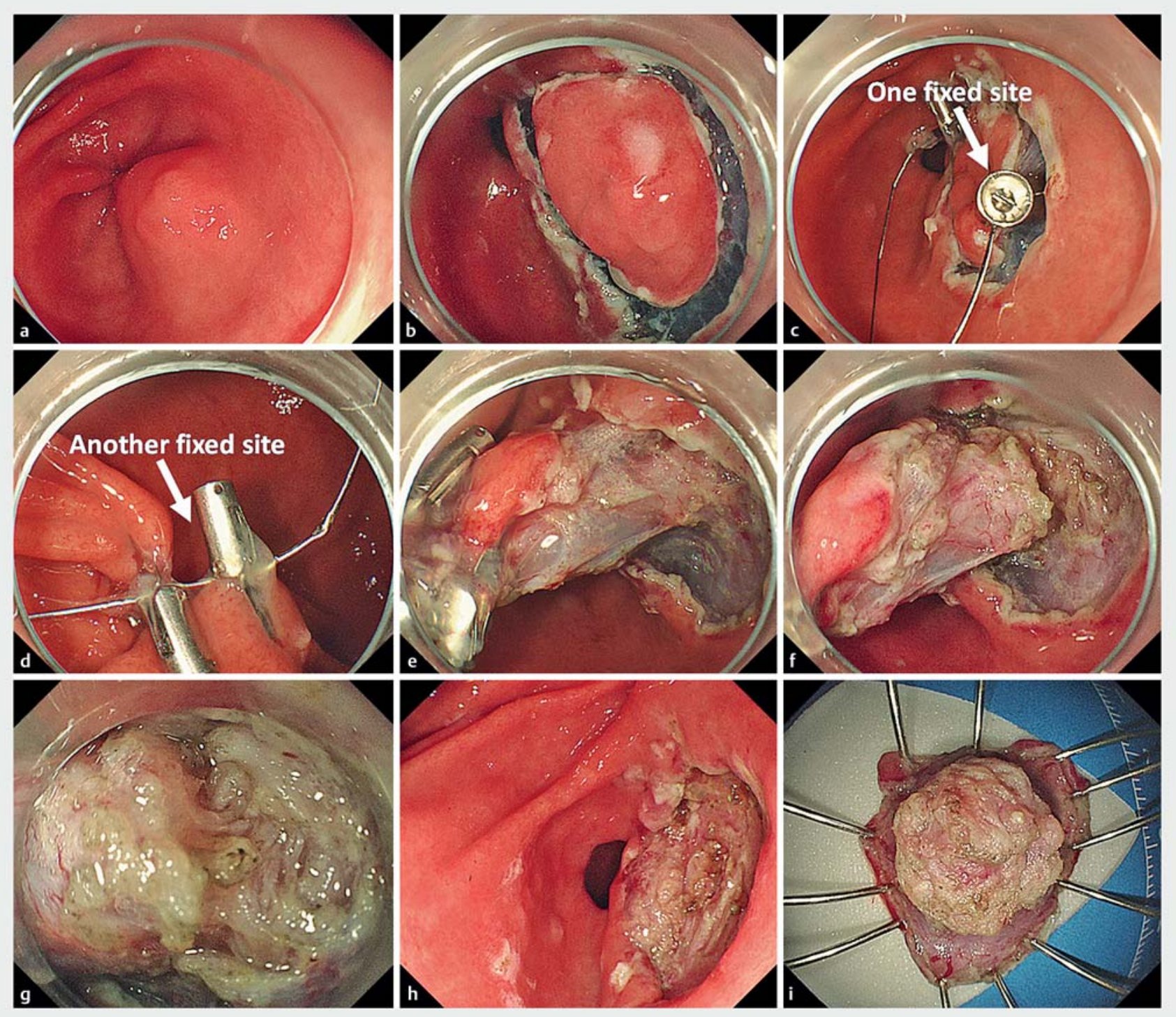

- Supplementary Fig. 3 ESD assisted by PSMT-SE for resection of a submucosal tumor at the posterior wall of the gastric antrum. Non-gastric mucosa-sparing peroral internal traction was used. The operation steps were the same as those described for $>$ Fig. 7 . 